\title{
Reviewers in this Issue
}

Prof. Khalilur Rahman

Department of Surgery

Enam Medical College \& Hospital, Savar, Dhaka

Prof. Deb Prasad Paul

Department of Surgery

Enam Medical College \& Hospital, Savar, Dhaka

Prof. Mithun Alamgir

Department of Community Medicine

Enam Medical College, Savar, Dhaka

Prof. Irin Perveen

Department of Gastroenterology

Enam Medical College \& Hospital, Savar, Dhaka

Prof. Sheuly Begum

Department of Obstetrics \& Gynaecology

Enam Medical College \& Hospital, Savar, Dhaka

Dr. Mahbub Hossain

Associate Professor

Department of Medicine

Enam Medical College \& Hospital, Savar, Dhaka

Dr. Haroon-or-Rashid

Associate Professor

Department of Pulmonology

Enam Medical College \& Hospital, Savar, Dhaka

Dr. Mohammad Quamrul Hasan

Associate Professor

Department of Gastroenterology

Enam Medical College \& Hospital, Savar, Dhaka

Dr. Lazina Sharmin

Former Associate Professor

Department of Paediatrics

Enam Medical College \& Hospital, Savar, Dhaka 